\title{
GEOGRAFÍA
}

\section{Geomorfología y peligros de origen natural en laderas aledañas a la localidad de Huallanca, provincia de Huaylas, departamento de Áncash}

RECIBIDO: $13 / 03 / 2017$

APROBADO: 04/12/2017

Juan Felipe Meléndez de la Cruz

Universidad Nacional Mayor de San Marcos

<jmelendezd@unmsm.edu.pe >

RESUMEN

La erosión hídrica y los deslizamientos son los dos procesos de mayor notoriedad en los interfluvios (laderas). El primero se refleja en laderas con cárcavas debido al impacto de las gotas de agua provenientes de las lluvias sobre una topografía muy inclinada. El segundo es el movimiento en masa del material superficial y que a su vez es muy erosivo porque es «cortante» e impacta fuertemente en los conos deyectivos de las cárcavas o en el talud de derrubios. La roca de basamento en este caso es un intrusivo que está muy fracturado y es poroso siendo otro factor favorable para la erosión.

Palabras Clave: Geoformas; litología; morfogénesis; geodinámica y peligros.

\section{Geomorphology and hazards of natural origin on slopes bordering the town of Huallanca, Province of Huaylas - Department of Ancash}

ABSTRACT

Water erosion and landslides are the two processes of greatest visibility in the interfluvios (slopes). The first one is reflected in slopes with gullies due to the impact of raindrops on a very sloping topography. The second is the mass movement of the surface material, which in turn is very erosive because it is "sharp" and strongly impacts the cones of the gullies or the slope of debris. The basement rock in this case is an intrusive that is very fractured and is porous being another favorable factor for erosion.

KEY wORD: Geoforms; lithology; morphogenesis; geodynamics and hazards 


\section{Introducción}

\section{Localización}

Desde el punto de vista geomorfológico, el área de estudio se localiza en los interfluvios (laderas) y en las terrazas de la margen izquierda del río Santa (aguas abajo) aledańas a la localidad de Huallanca.

Políticamente el área de estudio se encuentra en el distrito de Huallanca de la provincia de Huaylas, departamento de Áncash. Huallanca se localiza aproximadamente a $39 \mathrm{~km}$ de la ciudad de Caraz. El acceso que más utiliza la población es partiendo de la ciudad de Huaraz, pasando por Caraz, desde allí hasta la localidad de Huallanca. En el Anexo 1, se acompaña un gráfico con la localización del área de trabajo.

El área de trabajo se encuentra comprendida en el marco de las siguientes coordenadas UTM en zona 18 Sur:

$185896.00 \mathrm{~m} \mathrm{E} 9024376.00 \mathrm{~m} \mathrm{~S}$

$185906.00 \mathrm{~m} \mathrm{E} 9023368.00 \mathrm{~m} \mathrm{~S}$

$184467.00 \mathrm{~m} \mathrm{E} 9023356.00 \mathrm{~m} \mathrm{~S}$

$184456.00 \mathrm{~m} \mathrm{E} 9024373.00 \mathrm{~m} \mathrm{~S}$

\section{Planteamiento del problema}

La localidad de Huallanca se encuentra localizado en el piedemonte de montańas exponiéndose a ser impactados por deslizamientos (flujos de detritos). Por lo tanto, hace falta señalar las zonas con mayores niveles de peligros a fin de tomar las medidas preventivas y de mitigación o reducción de riesgos de desastres.

\section{Objetivo general}

El objetivo general del estudio es hacer una zonificación de peligros de origen natural por deslizamientos que puede impactar a la localidad de Huallanca. Previamente, se estudiará la geomorfología del área de trabajo.

\section{Justificación}

En el área no existen estudios geomorfológicos a escala grande que pueden ser utilizados para estimar los peligros de origen natural. El gobierno local requiere de estos trabajos para llevar a cabo una adecuada gestión de riesgos de desastres.

Asimismo, desde el punto de vista académico el área de estudio se debe convertir en un laboratorio de campo para estudiar los procesos de erosión en ladera, sobre todo la erosión hídrica y los deslizamientos (flujo de detritos).

\section{Material y métodos}

\subsection{Geología}

El área de estudio se emplaza sobre el batolito de la Cordillera Blanca cuya litología está compuesta de rocas granodioritas y tonalitas y que son de edad del terciario superior (esta litología comprende el basamento o roca madre). En el plano estructural esa litología tiene una foliación general y un juego bien desarrollado de diaclasas.

El mencionado batolito está cubierto de potentes capas de mantos de derrubios (arenas y clastos) resultado de antiguos deslizamientos (Foto 1). De acuerdo a la Hoja 18-h Corongo, de la Carta Geológica Nacional, estos materiales superficiales están clasificados como depósitos coluviales (INGEMMET 1995). El batolito aflora en las partes altas de laderas donde las pendientes son superiores a los $45^{\circ}$.

La edad de los materiales superficiales no es mayor a los 10,000 años y corresponden al cuaternario reciente (holoceno). Por otro lado, los depósitos aluvionales están localizados en las terrazas del río Santa compuestos de arcillas, arenas, clastos y bloques estriados y también corresponden al holoceno (Foto 11).

TABLA 1. GEOLOGÍA

\begin{tabular}{|l|l|l|l|}
\hline Formación o roca & Símbolo & Litología & Lugar donde aflora \\
\hline Depósitos coluviales & Qh-co & Arenas, clastos y bloques & $\begin{array}{l}\text { Corte de carretera de penetración aledaño a } \\
\text { Huallanca }\end{array}$ \\
\hline Depósitos aluvionales & Qh-al & Arcillas, arenas, cantos, bloques estriados & Terrazas contiguos a localidad de Huallanca \\
\hline Batolito de la Cordillera Blanca & Ts- gd/to & $\begin{array}{l}\text { Rocas intrusivas como la granodiorita y la } \\
\text { tonalita }\end{array}$ & $\begin{array}{l}\text { Caberas de cárcavas aledañas a localidad de } \\
\text { Huallanca }\end{array}$ \\
\hline
\end{tabular}




\subsection{Geomorfología}

\subsubsection{Geomorfología regional}

A nivel regional la unidad geomorfológica es la Cordillera Occidental caracterizado por una morfología de montańas. Los interfluvios del sector comprenden laderas de montaña y piedemonte que están siendo denudadas por procesos de erosión hídrica y deslizamientos de diferente intensidad (Foto Nro. 4).

Otra unidad importante es el valle del río Santa que a su vez están configurados por terrazas aluvionales. El río Santa en el sector de la localidad de Huallanca se encuentra localmente encańonadas sobre todo aguas arriba, en el paraje conocido con el nombre de «Cañón del Pato». En Huallanca, las terrazas aluvionales, del citado río, fueron rellenadas por antiguos aluviones, cuyos taludes se encuentran escarpados similar a los acantilados.

\subsubsection{Geomorfología local}

\section{Morfodinámica y análisis geomorfológico}

Los procesos de denudación que ocurren en los interfluvios (laderas) aledaños a la localidad de Huallanca son los deslizamientos o flujos de detritos. Esta erosión es relevante en los canales de las cárcavas que a su vez generan una erosión lineal. Esa erosión lineal consiste en una excavación del propio fondo del canal de la cárcava (Foto 8). Los flujos de detritos pueden impactar en los conos deyectivos de las cárcavas, y en parte están ocupadas por viviendas, se ha trazado de manera aproximada esas geoformas.

En ese sentido, la mayor intensidad de la erosión se localiza en los canales de cárcavas de mayor profundidad, situación que se ha utilizado como el principal indicador del mencionado proceso. Otro indicador para señalar zonas de mayor erosión son los sectores de las laderas con mayores pendientes. El último indicador de la intensidad de la erosión que se ha observado en el campo es la densidad de disección. La erosión hídrica concentrada ha disectado la roca densamente en algunas zonas (Foto 2). Normalmente, la distancia entre las disecciones de canales de cárcavas es de pocos decímetros (Foto 3).

Cabe agregar, que geológicamente la litología resultante de los deslizamientos están señalado como depósitos coluviales. Ese material es de edad del cuaternario reciente y es susceptible de ser erosionado por acción de la escorrentía. Es decir, el agua (gotas de agua) que se precipita e impacta sobre las laderas debido a la energía cinética. El mencionado impacto va originar erosión y va ir removiendo las partículas del material superficial de las laderas. Asimismo, hay que tener en cuenta que el Perú es un país sísmico, por encontrarse en el Cinturón de Fuego de Pacifico, por lo tanto, los sismos es otra variable que interviene en los procesos de erosión. En ese sentido, los agentes como el agua y el viento, así como los declives altos de las laderas (topografía), el material superficial susceptible a ser erosionado (litología), y los sismos como eventos de geodinámica interna son factores que condicionan la intensidad de los procesos gravitatorios o coluviales o de erosión.

\section{Mapa geomorfológico y clasificación geomorfológica}

Para llevar a cabo el estudio se preparó un mapa geomorfológico a escala 1: 5 000, ver Anexo 2, en base a una clasificación adaptada al objetivo del trabajo. Se ha seleccionado una clasificación de carácter morfogenético para facilitar la descripción y la explicación del origen las formas del relieve y los agentes o factores que lo modelan o erosionan.

Las formas del relieve del área de estudio se han clasificado considerando la aplicación de la geomorfología a los peligros de origen natural. Es decir, la identificación de zonas probables a ser impactados por deslizamientos o caídas. La mencionada clasificación está dividida en tres grandes grupos, la primera las geoformas originadas por procesos endógenos, la segunda las formas más vinculadas a los procesos de erosión. El tercer grupo son las formas construidas por el hombre para promover su seguridad física. Las formas del relieve son las siguientes:

- Formas endógenas: laderas con afloramientos de roca intrusiva

- Formas derivadas de procesos exógenos: mantos de derrubios consolidados, canal de cárcava, cabecera de cárcava, terraza aluvional (Río Santa), lecho menor del río.

- Formas construidas por el hombre: carretera, canaleta.

\subsubsection{Vegetación}

La vegetación encontrada en el área de estudio pertenece a la comunidad de las xerofitas, es decir cactáceas. Esta vegetación normalmente se desarrolla en medio semiárido donde la precipitación es escasa en gran parte del año (Foto 7). 
Se ha encontrado en las laderas que se ha plantado especies vegetales con el fin de proteger el suelo de la erosión, sin embargo, no se han desarrollado probablemente por falta de agua.

\section{Resultados}

\subsection{Tipos de peligros de origen natural}

Los tipos de peligros de origen natural que está expuesta la población de Huallanca es a ser impactados por deslizamientos (flujos de detritos) de manera canalizada y no canalizada y las caídas. El flujo canalizado se refiere al desplazamiento de los detritos por los canales de las cárcavas debido a procesos gravitatorios y lluvias que a su vez va excavando su fondo. El proceso termina cuando impacta y a su vez deposita el referido material superficial en los conos deyectivos. El flujo no canalizado se da en forma no concentrada por las laderas impactando y depositando al pie de ellas, formando los taludes de derrubios.

Las caídas de rocas es otro tipo de peligro, que está expuesta la población, pudiendo ocurrir durante sismos sobre todo en las laderas de altas pendientes. Los bloques que tienen diámetros mayores a 1 metro pueden perder inercia y desplazarse ladera abajo e impactar las viviendas.

\subsection{Metodología para analizar los peligros de origen natural}

\section{Zonificación de peligros}

Se ha utilizado la capa "mapa geomorfológico» como base para hacer una zonificación de peligros.
Las mencionadas zonas de peligro se han delimitado considerando la clasificación geomorfológica señalada anteriormente. Es decir, las «cabeceras de cárcavas», "Conos deyectivos», «mantos de derrubios consolidados», etc.

\section{Modelo para estimación de los niveles de peligros}

Teniendo en cuenta la información obtenida en gabinete y campo se llevó a cabo un análisis geomorfológico obteniendo como resultado el siguiente modelo para medir la intensidad de los peligros.

\section{Valoración de Indicadores seleccionados}

Se ha elegido los siguientes indicadores o variables para medir la erosión:

- Profundidad de erosión (cárcava o disección),

- Pendientes del terreno y

- Densidad de disección.

Para el caso de las pendientes del terreno se ha considerado que los deslizamientos pueden ocurrir con mayor intensidad en laderas con pendientes mayores a $45^{\circ}$.

La profundidad de erosión se refiere a la profundidad del canal de la cárcava. Los "pesos» que se han señalado tienen el objetivo de identificar los espacios más probables de ocurrir deslizamientos. En la tabla 2 se observa la manera cómo se diseñó el modelo.

Valoración de cada indicador o variable

Son los «pesos» que corresponden a cada uno de los indicadores seleccionados para medir la intensidad

TABLA 2. MODELO PARA ESTIMAR LOS NIVELES DE PELIGROS

\begin{tabular}{|c|c|c|c|c|c|c|c|}
\hline $\begin{array}{c}\text { Zona } \\
\text { Nro }\end{array}$ & Nombre de la zona & $\begin{array}{l}\text { Profund. } \\
\text { erosión }(A)\end{array}$ & \begin{tabular}{|c|}
$\begin{array}{c}\text { Pendientes del } \\
\text { terreno }(\mathrm{B})\end{array}$ \\
\end{tabular} & Disección $(\mathrm{C})$ & Valor Total & Ponderación de 0 a 1 & $\begin{array}{l}\text { Nivel de } \\
\text { peligro }\end{array}$ \\
\hline 1 & Cono deyectivo & & & & & $(A+B+C) / 12$ & \\
\hline II & Talud de derrubios & & & & & $(A+B+C) / 12$ & \\
\hline III & Terraza aluvional & & & & & $(A+B+C) / 12$ & \\
\hline IV & Talud de derrubios & & & & & $(A+B+C) / 12$ & \\
\hline V & Talud de derrubios & & & & & $(A+B+C) / 12$ & \\
\hline $\mathrm{VI}$ & Cono deyectivo & & & & & $(A+B+C) / 12$ & \\
\hline VII & Cono deyectivo & & & & & $(A+B+C) / 12$ & \\
\hline \multirow[t]{2}{*}{ VIII } & Cono deyectivo & & & & & $(A+B+C) / 12$ & \\
\hline & Valor máximo & 4 & 4 & 4 & $\begin{array}{c}12 \text { (suma de } \\
\text { valores max.) }\end{array}$ & & \\
\hline
\end{tabular}


del proceso de erosión. Se ha preparado las siguientes tablas adecuadas a la realidad del área de estudio.

TABLA 3. PROFUNDIDAD DE EROSIÓN (CÁRCAVA O DISECCIÓN)

\begin{tabular}{|c|c|}
\hline Rango en metros & Valor \\
\hline $10-5$ & 4 \\
\hline $5-2$ & 3 \\
\hline $2-1$ & 2 \\
\hline$<1$ & 1 \\
\hline
\end{tabular}

TABLA 4. PENDIENTES DEL TERRENO

\begin{tabular}{|c|c|}
\hline Rango & Valor \\
\hline$>45^{\circ}$ & 4 \\
\hline $45^{\circ}-40^{\circ}$ & 3 \\
\hline$<0^{\circ}-24^{\circ}$ & 2 \\
\hline menores a $24^{\circ}$ & 1 \\
\hline
\end{tabular}

TABLA 5. DISTANCIA ENTRE CÁRCAVAS (DENSIDAD DE DISECCIÓN)

\begin{tabular}{|c|c|}
\hline Rango en metros & Valor \\
\hline$<10$ & 4 \\
\hline $10-20$ & 3 \\
\hline $20-50$ & 2 \\
\hline$>50$ & 1 \\
\hline
\end{tabular}

\section{Discusión de resultados}

\section{Estimación de peligros}

Peligro o amenazas es la probabilidad que un fenómeno de origen natural pueda impactar a la población, la infraestructura física o las unidades productivas de la sociedad. En la práctica, un estudio de esa naturaleza consiste en una «zonificación de peligros» con diferentes niveles de peligros. En este caso, como se señaló anteriormente, el tipo de peligros es geológicogeomorfológico (deslizamientos y caídas). Sin embargo, también influyen las variables climáticas como la temperatura, la precipitación, incluso el viento, en los procesos de erosión hídrica y deslizamientos. Es decir, las laderas cubiertas de espesos mantos de derrubios durante miles de años están expuestos a los intensos procesos de meteorización y llega un momento en que se produce la erosión con desplazamiento cortante mediante los flujos de detritos. Es decir, los deslizamientos son de tipo traslacional ladera abajo.

Para estudiar los peligros del área de estudio se tomó en cuenta los resultados alcanzados en análisis geomorfológico que consistió primero en identificar las formas del relieve que se plasmó en un mapa geomorfológico. Segundo, se utilizó los resultados alcanzados en la parte de la morfodinámica. Tercero, se diseñó un modelo para estimar las zonas de peligros. Se empleó como apoyo los Sistemas de Información Geográfica (SIG) para analizar la información geoespacial y poner en práctica el mencionado modelo con el fin de estratificar los peligros en el área de estudio.

Los niveles de peligros se midieron de la siguiente manera: Niveles Muy alto, los valores mayores a 0.75; Nivel Alto, entre 0.75 a 0.50 y Nivel Medio entre 0.49 a 0.25 . Los mencionados niveles de riesgos han sido empleados por el Instituto Nacional de Defensa Civil (INDECI) particularmente en los proyectos de Ciudades Sostenibles. Las delimitaciones de las zonas de peligros están basadas en las formas de relieve como componente de soporte espacial. El resultado de los niveles de peligros encontrados en el área de estudio se muestra en la Tabla 6:

Cabe indicar que la zona Nro. 3 «terraza aluvional del río Santa» está expuesta a la erosión fluvial durante crecidas. Mientras que en las zonas restantes por estar localizados al pie de laderas están expuestas a deslizamientos y caídas. En el Anexo 3 se presentan el mapa de Zonificación de peligros. Asimismo, en el

TABLA 6. RESULTADOS DE LOS NIVELES DE PELIGROS POR ZONAS

\begin{tabular}{|l|l|c|c|c|c|c|c|}
\hline Zona N. & Nombre de la zona & $\begin{array}{c}\text { Profundidad de } \\
\text { erosión }\end{array}$ & $\begin{array}{c}\text { Pendientes del } \\
\text { terreno }\end{array}$ & $\begin{array}{c}\text { Densidad } \\
\text { disección }\end{array}$ & $\begin{array}{c}\text { Valor total } \\
\text { Ponderación } \\
\text { de } 0 \text { a } 1\end{array}$ & $\begin{array}{c}\text { Nivel de } \\
\text { peligro }\end{array}$ \\
\hline I & Cono deyectivo & 2 & 2 & 2 & 6 & 0.5 & Alta \\
\hline II & Talud de derrumbes & 1 & 3 & 3 & 7 & 0.58 & Alta \\
\hline III & Terraza aluvional del río Santa & 4 & 4 & 1 & 9 & 0.75 & Alta \\
\hline IV & Talud de derrubios & 2 & 3 & 3 & 8 & 0.67 & Alta \\
\hline V & Talud de derrubios & 1 & 4 & 3 & 8 & 0.67 & Alta \\
\hline VI & Cono deyectivo & 4 & 3 & 2 & 9 & 0.75 & Alta \\
\hline VII & Cono deyectivo & 1 & 3 & 2 & 6 & 0.5 & Alta \\
\hline VIII & Cono deyectivo & 4 & 3 & 1 & 8 & 0.67 & Alta \\
\hline
\end{tabular}


Anexo 4 se presentan las fotos. Las fotografías muestran las geoformas y los principales procesos geomórficos que se desarrollan en el área de estudio.

Si bien es cierto la totalidad del área de estudio se encuentra en situación de peligro alto a ser impactos sobre todo por deslizamientos. Se ha encontrado medidas de mitigación que pueden controlar o disminuir la intensidad de los peligros de origen natural. Si se toma en cuenta esas medidas de mitigación los niveles de peligros podrían reducirse, hasta un nivel medio.

Es decir, que esta investigación ha tomado exclusivamente las variables de origen natural. En un eventual estudios de riesgos, la disminución de la vulnerabilidad de la población estaría sujeta al mantenimiento de las medidas de mitigación. En ese sentido, resulta de suma importancia el mantenimiento de las canaletas y si es posible la arborización de las laderas a fin de disminuir los niveles de vulnerabilidad de la población de Huallanca.

Los riesgos son dinámicos y pueden variar de un año a otro, inclusos en meses, depende bastante de la gestión local de los riesgos de desastres. Este trabajo contribuye con la información espacial de peligros para considerarlo como un antecedente en el proceso de gestión de riesgos de desastres. Por esa razón, es recomendable realizar estudios de riesgos anualmente y las propuestas de gestión deben ser difundidas a la población a fin de prevenir nuevos escenarios de riesgos. Asimismo, el gobierno local debería disponer de una base de datos geológico-geomorfológica y peligros para facilitar la actualización permanente de los estudios de riesgos.

\section{Conclusiones}

1. Lo más notorio de la geomorfología estudiada es la erosión lineal que ocurren en los talwegs de las cárcavas. Este proceso de erosión consiste en excavar el fondo de las cárcavas cortando un material superficial muy arenoso y con clastos.

2. Los citados procesos de erosión corresponden a deslizamientos (flujos de detritos) en medios semi-áridos. En los climas semi-áridos se pueden desarrollar los mayores procesos de erosión sobre todo si se dan las condiciones geológicageomorfológicas, como es el presente caso de geomorfología aplicada.
3. Las topografías de las laderas comprenden declives entre $45^{\circ}$ a 24 , encontrándose localmente afloramientos de roca madre. Esos declives de laderas es uno de los factores determinantes de los procesos de erosión.

4. Asimismo, la profundidad y densidad de las cárcavas son indicadores de que los deslizamientos son intensos debido a la acción erosiva y muy cortante que ocurre sobre las laderas.

5. La población asentada en las zonas (6 y 8) están expuestas al impactado por deslizamientos (flujo de detritos) por estar localizados en una zona de piedemonte. Es decir, se ubican en el "pie» de laderas que en su parte alta normalmente tienen pendientes escarpadas a inclinadas.

6. Asimismo, las mayores intensidades de los procesos de erosión en las laderas están relacionados con fuertes precipitaciones pluviales que pueden ocurrir en verano. Por lo tanto, si se presentaran lluvias intensas de varias horas, los deslizamientos serían intensos y podrían impactar a las viviendas que se ubican en los conos deyectivos de las cárcavas y en los taludes de derrubios.

7. Se ha encontrado canaletas que cortan transversalmente las laderas y que es una buena medida de mitigación para reducir el impacto de los deslizamientos, sin embargo, se observó una falta de mantenimiento porque están colmatados.

8. Otra parte de la población tiende a localizarse sobre la terraza aluvional del río Santa exponiéndose a los derrumbes (caída de viviendas) por la erosión fluvial durante probables crecidas de la mencionada corriente. Es decir, en eventos de máximas crecidas del río Santa podrían ocurrir socavamiento de la base de las terrazas aluvionales por erosión fluvial y originar derrumbes de parte de esta geoforma.

9. Esta investigación es uno de los casos típicos de las aplicaciones de la geomorfología a los peligros de origen natural. Se trata de un caso de una geomorfología «urbana» o ambiental porque al estudiar las formas del relieve, se ha observado la «mano» de la sociedad porque lo ha intervenido.

10. Por otro lado, la investigación es un caso de riesgos de desastres, de "causa-efecto». Por un lado, las causas de los peligros son de origen natural y comprenden procesos de movimientos en masa en laderas. El efecto comprende las formas del relieve o geoformas (conos deyectivos de las cárcavas y 
taludes de derrubios) que pueden ser impactados por los delizamientos o caídas. Esas geoformas son espacios naturales de acumulaciones de material erosionado, sin embargo, han sido urbanizados.

\section{Referencias bibliográficas}

Carenas, María; Giner, Jorge (2014). Geología. Madrid: Lavel Industria Gráfica.

Cenepred (2015). Manual para la evaluación de riesgos originados por fenómenos naturales. Lima.

Dávila, Jorge (2011). Diccionario geológico. Lima.

De Pedraza, Javier (1996). Geomorfología. Principios, métodos y aplicaciones. Madrid: Editorial Rueda.
Instituto Geológico Minero y Metalúrgico (2015). Informe técnico $N^{\circ}$ A 6680 - Evaluación geodinámica de los flujos de detritos del 23/03/2015 entre las quebradas Rayos del sol y Quirio (Chosica) y Cashahuacra (Santa Eulalia) - Provincias Lima y Huarochiri. Lima

Instituto Geológico Minero y Metalúrgico (1995). «Geología de los cuadrángulos de Pallasca, Tayabamba, Corongo, Pomabamba, Carhuaz y Huari». Boletín 60. Lima.

Lugo Hup, José (1989). Diccionario geomorfológico. México: UNAM.

Meléndez, J., Pérez, W., Osejo, J. (2011). Guía geomorfológica. Primera aproximación. Lima: Sociedad Geográfica de Lima y el Colegio de Geógrafos del Perú.

Ministerio del Medio Ambiente (2004). Guia para la elaboración de estudios del medio físico. España.

\section{ANEXO 1. MAPA DE LOCALIZACIÓN}
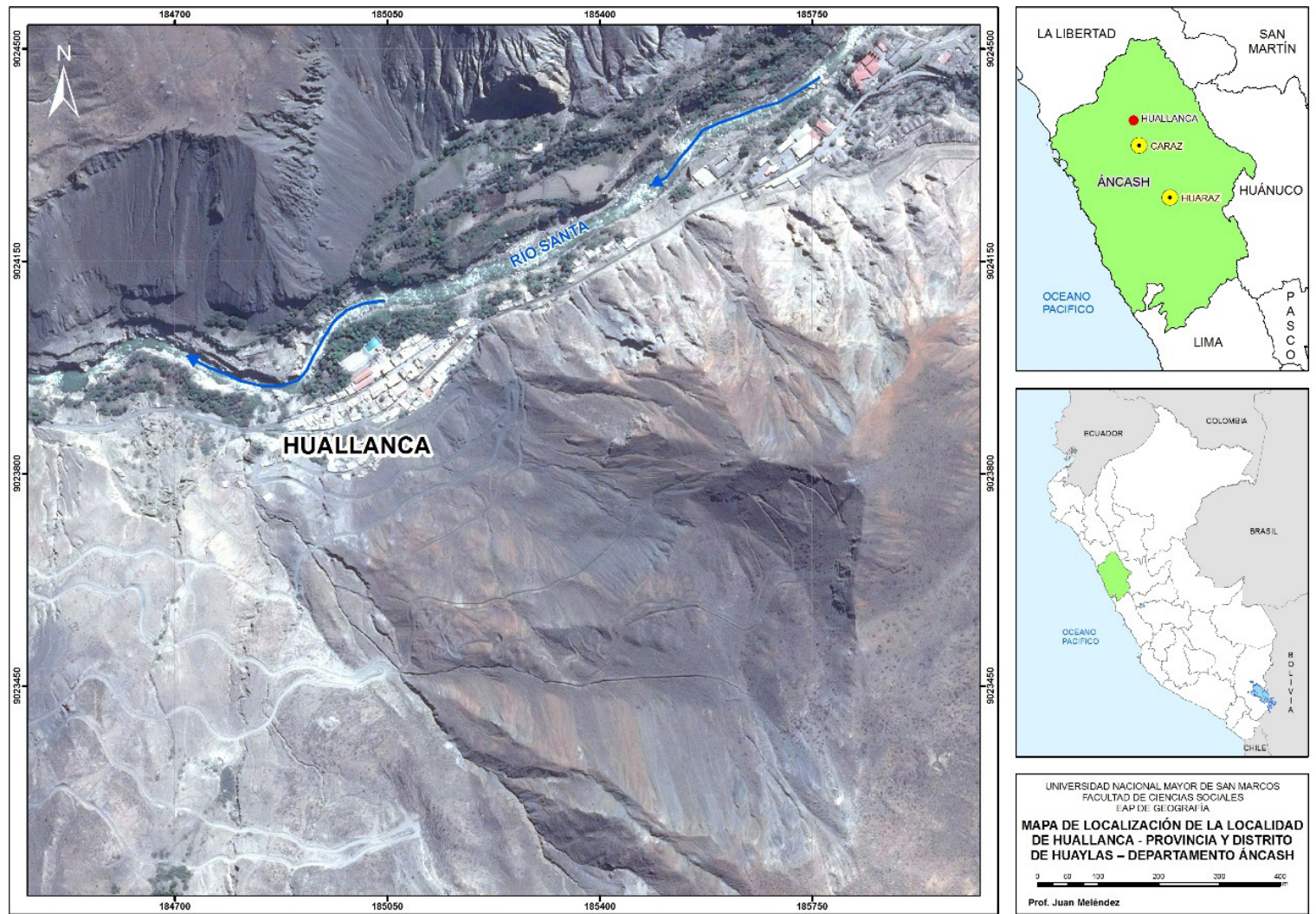
ANEXO 2. MAPA GEOMORFOLÓGICO

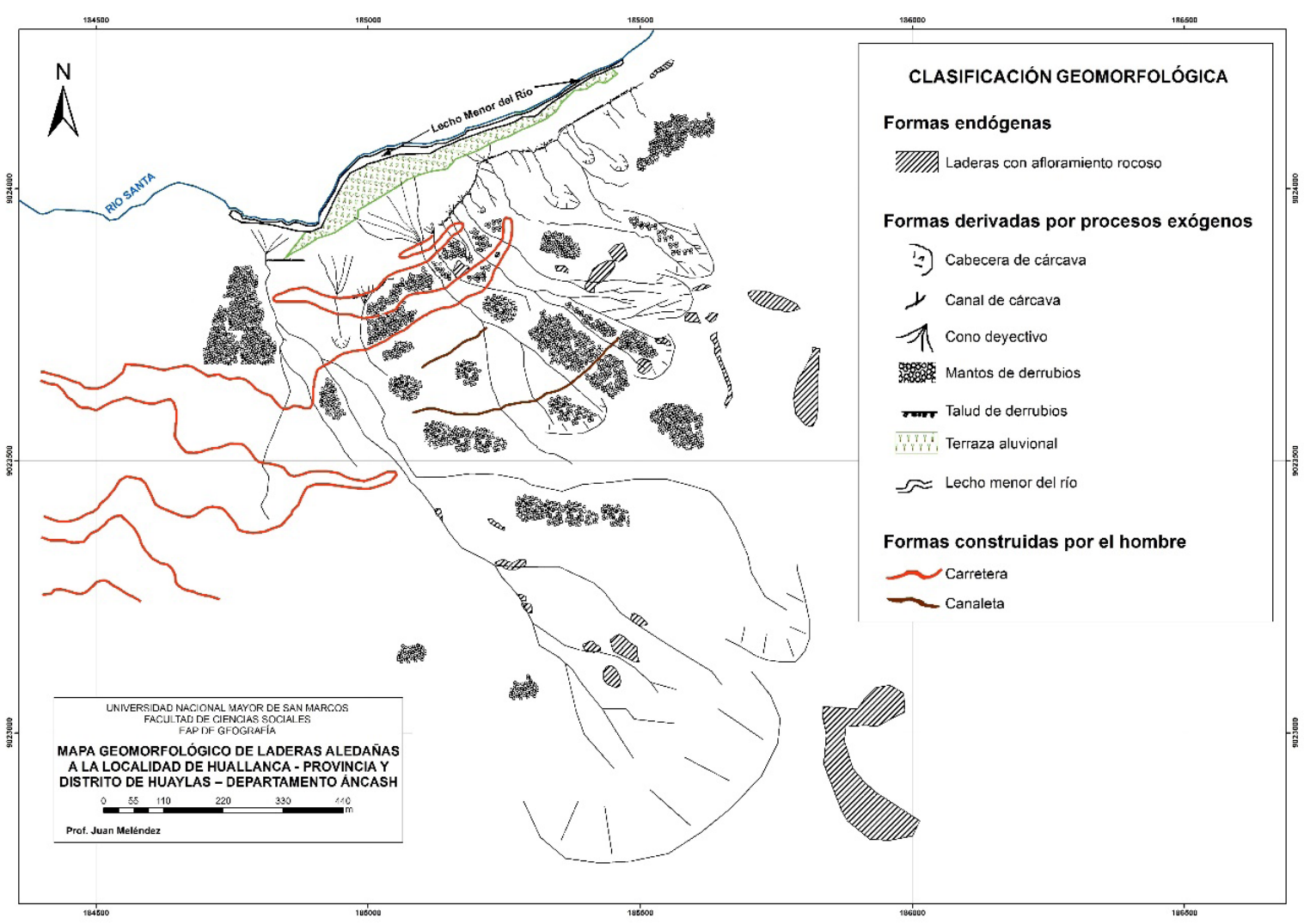

ANEXO 3. MAPA «ZONIFICACIÓN DE PELIGROS»

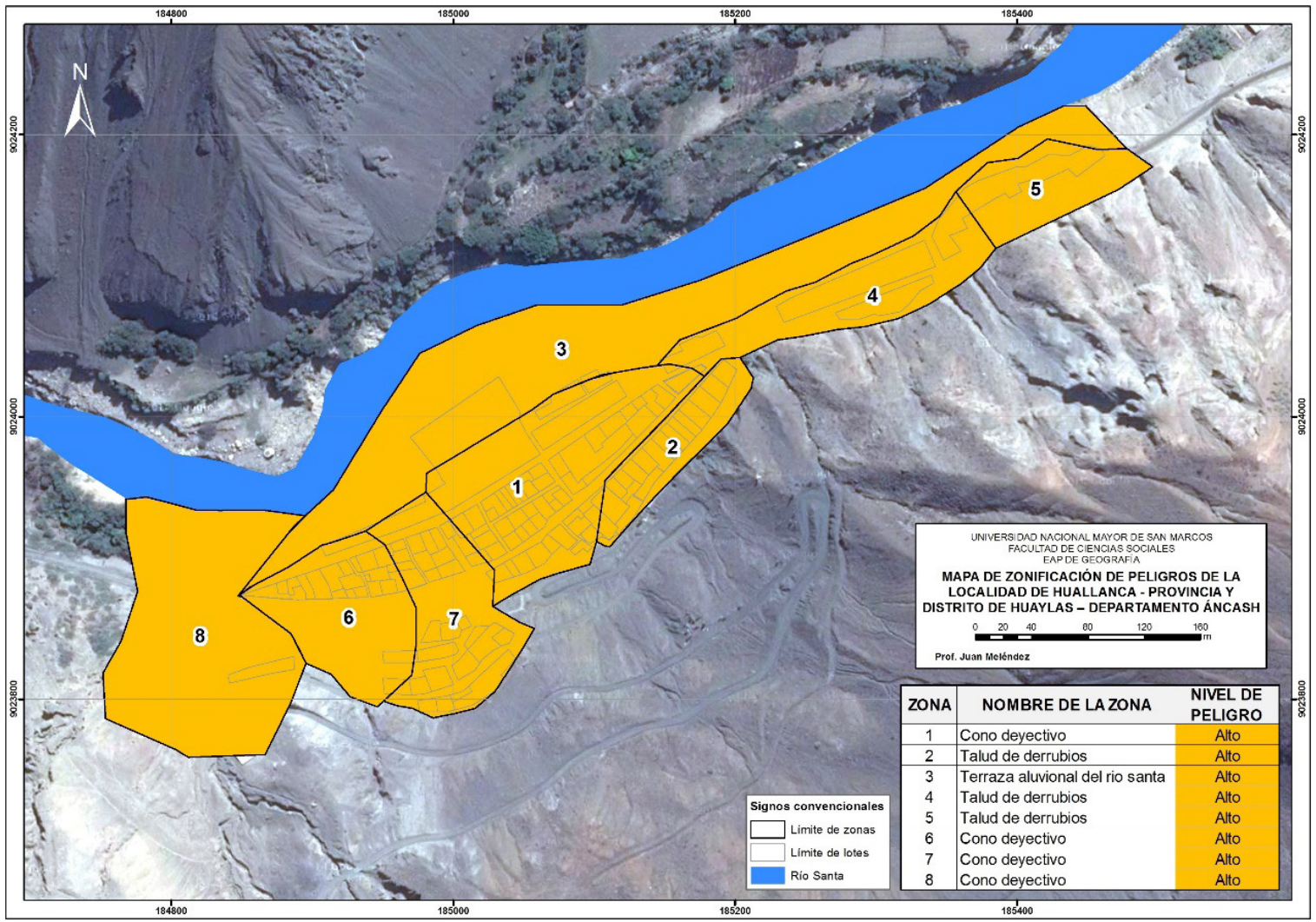


ANEXO 4. FOTOS
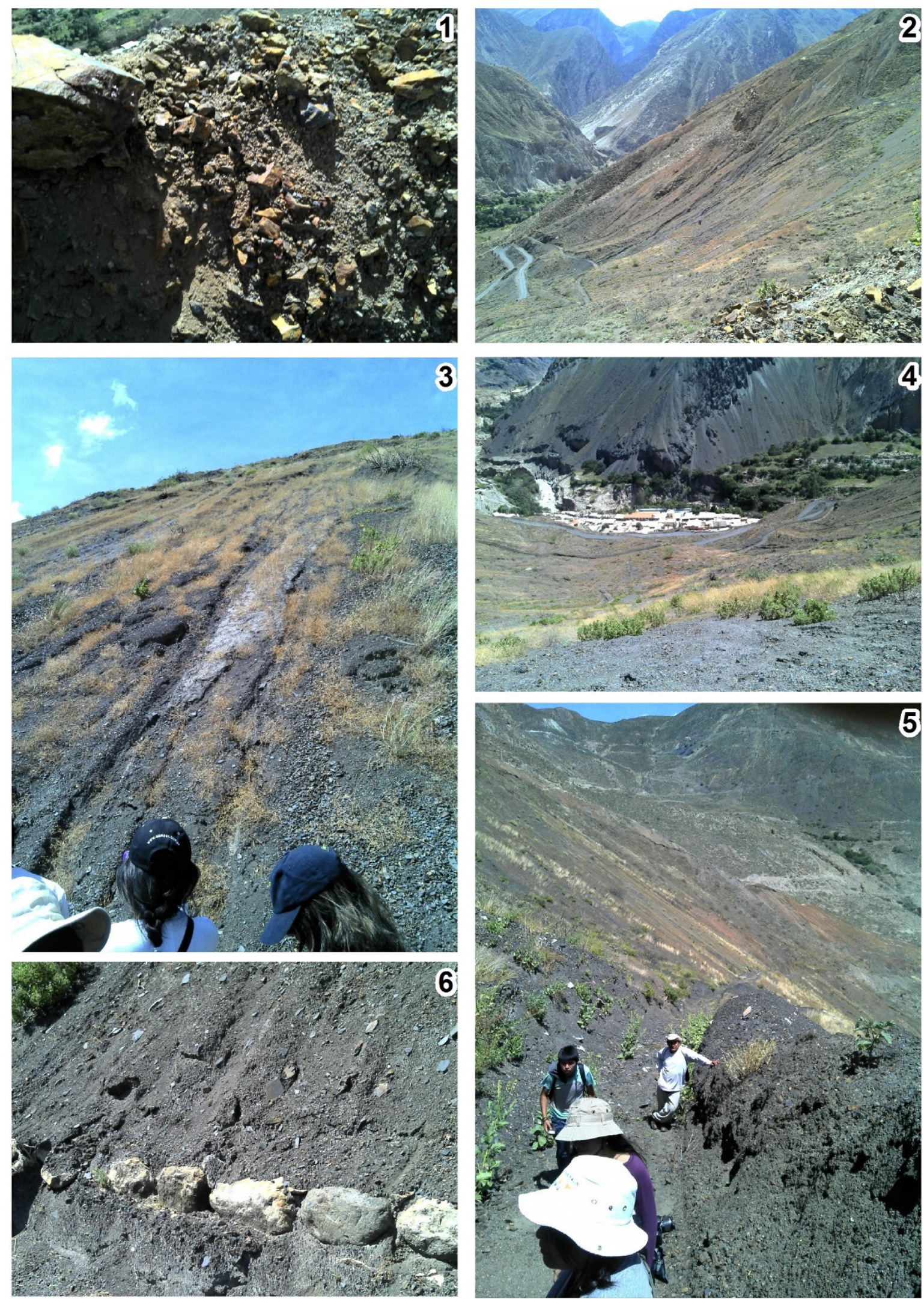

1. Material superficial. 2. Disección (laderas). 3. Distancia entre disecciones. 4. Interfluvios (laderas). 5. Canaleta. 6. Canaleta colmatada. 

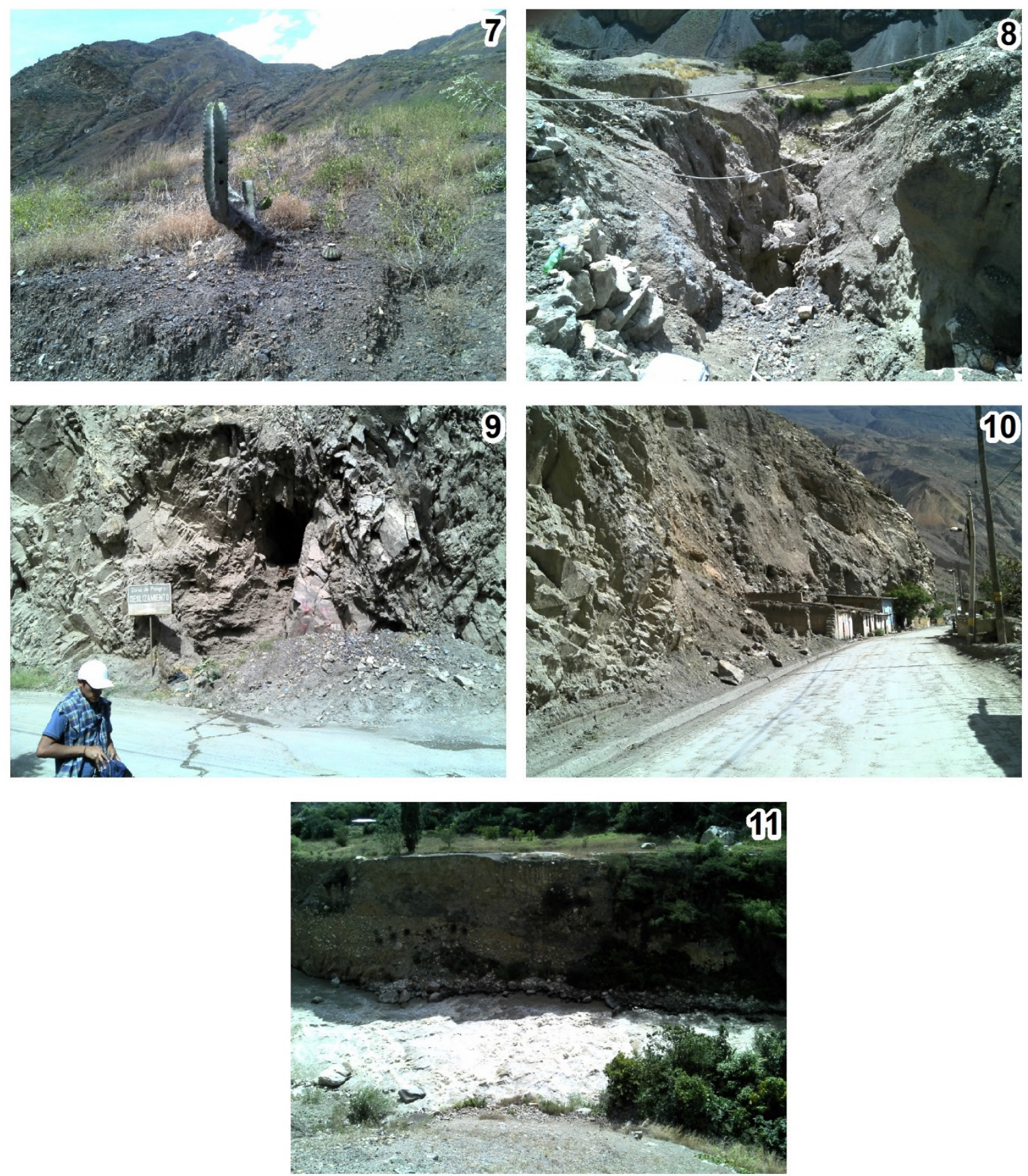

7. Vegetación xerofita. 8. Canal de cárcava. 9. Afloramiento de roca. 10. Caídas de rocas. 11. Terraza aluvial río Santa. 